\title{
Submitted: Validity of measurements of testicular volume obtained Accepted: 10.08.2020 Published: by a built-in software of ultrasound systems: with formula recommended by updated guidelines as reference
} 28.09.2020

Keywords testis, measurement, formula, ultrasound

\author{
Dongyan Cai, Size Wu, Ya Li, Qingfang Chen \\ Department of Ultrasound, The First Affiliated Hospital of Hainan Medical University, \\ Haikou, China \\ Correspondence: Size Wu, Department of Ultrasound, The First Affiliated Hospital of Hainan \\ Medical University, No.31, Longhua Road, Haikou 570102, China; tel.: 86-0898-66774347, \\ e-mail:wsz074@aliyun.com
}

DOI: $10.15557 /$ JoU.2020.0030

\begin{abstract}
Aim: To investigate the validity of measurement of testicular volume acquired by a built-in software in different ultrasound systems with reference to the updated guidelines. Materials and methods: Archives of 1,976 patients who had undergone scrotal ultrasound evaluation were reviewed. A total of 973 patients with 1,909 testes, who had undergone ultrasound measurement of the testicular volume, were included in the study, and 1,003 patients were excluded. The age of enrolled patients ranged from 17 to 66 years (median age of 39 years). The ultrasound systems included Siemens Sonoline S2000, Philips EPIQ5, GE Logiq E9, Hitachi Aloka prosounda7, Mindray DC-8 and Mindray Resona7. The transducers have imaging frequencies of 5-14 MHz. Validity of the measurement of testicular volume acquired by a built-in software in different ultrasound systems was assessed with reference to the formula that Volume $(\mathrm{V})=$ Length $(\mathrm{L}) \times$ Width $(\mathrm{W}) \times$ Height $(\mathrm{H}) \times 0.71$, recommended by the updated guidelines, by recalculating the original numbers using a calculator. Results: The values obtained by the built-in software of Mindray DC-8 and Mindray Resona7 ultrasound systems and measurements recalculated on a computer were all in concordance; and the values obtained by the built-in software of Siemens Sonoline S2000, Philips EPIQ5, GE Logiq E9, and Hitachi Aloka prosound 7 ultrasound systems and measurements recalculated on computer were all discordant. The same testicular measurements calculated with different formulas $(\mathrm{V}=\mathrm{L} \times \mathrm{W} \times \mathrm{H} \times 0.71$ vs. $\mathrm{V}=\mathrm{L} \times \mathrm{W} \times \mathrm{H} \times 0.52)$ produced $26.76 \%$ difference. Conclusion: Values of testicular volume obtained by some ultrasound systems are not accurate with reference to the formula recommended by the updated guidelines.
\end{abstract}

\section{Introduction}

In reproductive medicine, andrology and urology, the measurement of testicular volume becomes a routine examination for infertile males, for it is generally regarded as an index representative of spermatogenesis or semen profile; in other male patients with gonadal anomalies, the measurement of testicular volume is a necessity, so accurate measurement of testicular volume is important in the evaluation of disease and may also help in determining proper treatment ${ }^{(1-3)}$. There are several methods available for the measurement of testicular volume, such as caliper, various orchidometers, medical imaging; among them, ultrasound examination is considered the most accurate ${ }^{(4-16)}$. Currently, ultrasound imaging becomes widely accessible and available, with other methods of measurement of testicular volume being almost replaced by ultrasound ${ }^{(7-16)}$. In clinical practice, the value of testicular volume is usually worked out immediately by a built-in software of an ultrasound system based on the measurements of testicular length, width and height (anteroposterior depth), and rarely implemented by manual calculation using a certain formula. Radiologists and urologists rarely suspect the quality and functions of advanced medical ultrasound systems 


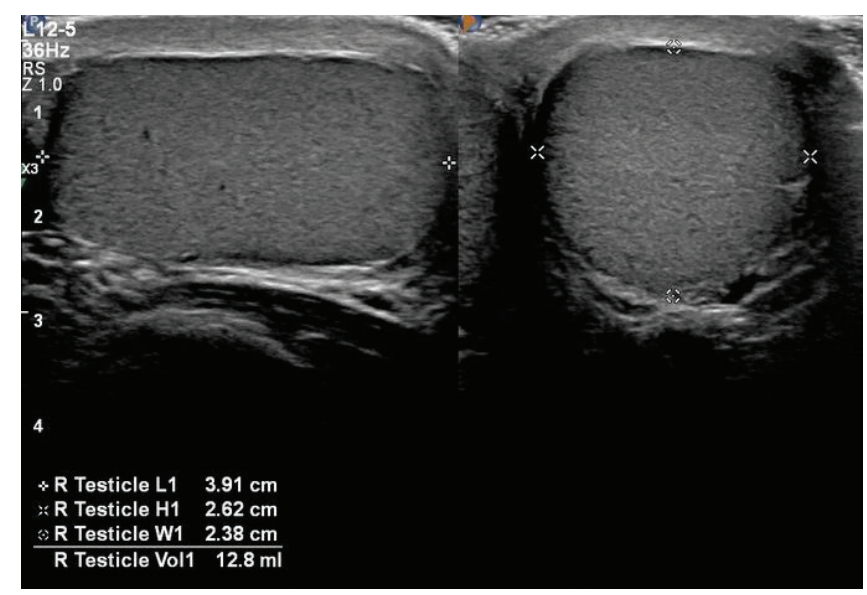

Fig. 1. A 31 year-old man with infertility. Measurement of the right testicular volume using ultrasound. The length, width, and height of the testis are $3.91 \mathrm{~cm}, 2.62 \mathrm{~cm}$, and $2.38 \mathrm{~cm}$, respectively; and the testicular volume is $12.8 \mathrm{~mL}$, which is obtained automatically by the Philips EPIQ5 ultrasound system with a built-in software

produced by world's well-known corporations, and the measurement of testicular volume by a built-in software of an ultrasound system has been trusted. However, every rule has its exception. The objective of this study was to investigate the validity of measurement of testicular volume acquired by a built-in software in different ultrasound systems with reference to the updated guidelines.

\section{Materials and methods}

Archives of 1,976 patients who had undergone consecutively scrotal ultrasound evaluation in a tertiary hospital with provincial human reproductive center from January 1, 2016 to January 1, 2020 were reviewed. A total of 973 patients with 1,909 testes, who had undergone ultrasound measurement of the testicular volume were included, and 1,003 patients who had not undergone measurement of the testicular volume were excluded. The age of enrolled patients ranged from 17 to 66 years (the median age was 39 years). The measurements of each testis saved in the Picture Archiving and Communication Systems (PACS) were reviewed and recalculated on a built-in calculator in the computer, with the formula that Volume $(\mathrm{V})=$ Length $(\mathrm{L}) \times$ Width $(\mathrm{W}) \times$ Height $(\mathrm{H}) \times 0.71$ used as a reference criterion, which has been proved to be the most accurate formula by previous studies and was recommended in Guidelines of the European Society of Urogenital Radiology Scrotal and Penile Imaging Working Group, and by the Guideline developed in collaboration with the American College of Radiology; Society for Pediatric Radiology; Society of Radiologists in Ultrasound ${ }^{(6-9,14-18)}$. For validity, the original values obtained by the built-in software of ultrasound systems were compared with the recalculated values. Meanwhile, some of the data obtained by different ultrasound systems were also validated using a formula that $\mathrm{V}=\mathrm{L} \times \mathrm{W} \times \mathrm{H} \times 0.52^{(7,9,10,17,18)}$. The protocols for the scrotal assessment of ultrasound were addressed in the following manner. The patient was examined in a supine recumbent position, after proper exposure of the scrotum, adequate volume of couple gel was placed onto the scrotum; a linear-array transducer was used to scan the scrotum and multiple parameters were assessed, including testicular volume (size), anatomic variants, and abnormalities of the testes, epididymides, tunica vaginalis and scrotal wall. Color Doppler flow imaging and pulsed wave Doppler assessment were performed as an integral part of the examination to evaluate vasculature in the testis and the spermatic cord. The transducer was placed on the scrotum without exerting pressure on the testis, and optimal dimensions were rendered to measure length, width and height (anteroposterior depth) of the testis. The linear-array transducers equipped in different ultrasound systems have imaging frequencies of 5-14 MHz. For each examination, the examination mode was preset to "small parts and testis"; parameters of gain, depth gain compensate, focus, depth, and scale were adjusted to an adequate status; the testicular volume was calculated automatically by the built-in software of the ultrasound system based on the measurement of the testicular length, width, and height. Fig. 1 illustrates the measurement of testicular volume. Images of duplex ultrasound imaging with and without measurements were saved in the PACS. The ultrasound systems used during this period included Siemens Acuson S2000 (Siemens Medical Systems, Inc., Ultrasound Group, Mountain View, California, USA); Philips EPIQ5 (Philips Ultrasound, Inc., Bothell, Washington, USA); GE Logiq E9 (GE Healthcare, Waukesha, WI, USA); Mindray DC-8 (Shenzhen Mindray Bio-Medical Electronics Co., Ltd., Shenzhen, China); Mindray Resona 7 (Shenzhen Mindray Bio-Medical Electronics Co., Ltd., Shenzhen, China); Hitachi-Aloka prosounda7 (Hitachi Aloka Medical Systems, Tokyo, Japan). Distribution of testicular volume measurements performed by different ultrasound systems was allotted.

\section{Ethical statements}

All procedures followed were in accordance with the ethical standards of the responsible committee on human experimentation (institutional and national) and with the World Medical Association Declaration of Helsinki (revised in 2000). The study was approved by the institutional review board of The First Affiliated Hospital of Hainan Medical University, and informed consent was waived due to the retrospective design.

\section{Results}

Distribution of testicular measurements by different ultrasound systems and outcome of validity were listed in Tab. 1 . Taking the formula that $\mathrm{V}=\mathrm{L} \times \mathrm{W} \times \mathrm{H} \times 0.71$ as the standard criterion, the values obtained by the built-in software of Mindray DC-8 and Mindray Resona 7 ultrasound systems and measurements recalculated on a computer were all concordant, and the values obtained by the built-in software of Siemens Acuson S2000, Philips EPIQ5, GE Logiq E9, and Hitachi Aloka prosounda7 ultrasound systems 
Tab. 1. Comparison of values obtained by the built-in software of ultrasound systems and re-calculated values with reference to the updated guidelines

\begin{tabular}{|c|c|c|c|c|c|}
\hline Ultrasound systems & Manufacture date & $\begin{array}{c}\text { Software } \\
\text { update date }\end{array}$ & $\begin{array}{c}\text { Distribution of testicular } \\
\text { measurement } \\
(\boldsymbol{n}=\mathbf{1 9 0 9 )}\end{array}$ & $\begin{array}{c}\text { Concordance } \\
\text { after validity } \\
(\boldsymbol{n}=\mathbf{5 6 6})\end{array}$ & $\begin{array}{c}\text { Discordance } \\
\text { after validity } \\
(\boldsymbol{n}=\mathbf{1 3 4 3})\end{array}$ \\
\hline Siemens Acuson S2000 & September 2009 & July 2015 & 352 & 0 & 352 \\
\hline Philips EPIQ5 & February 2018 & No & 224 & 0 & 224 \\
\hline GE Logiq E9 & March 2017 & No & 362 & 363 & 0 \\
\hline Mindray DC-8 & June 2012 & January 2017 & 363 & 203 & 0 \\
\hline Mindray Resona 7 & November 2017 & No & 203 & 0 & \\
\hline $\begin{array}{c}\text { Hitachi Aloka } \\
\text { Prosound a7 }\end{array}$ & October 2010 & No & 505 & & 505 \\
\hline
\end{tabular}

and measurements recalculated on computer were all discordant. The overall conformity rate was $29.65 \%$. If the formula that $\mathrm{V}=\mathrm{L} \times \mathrm{W} \times \mathrm{H} \times 0.52$ was used as the standard criterion, the above results of concordance and discordance for value comparison reversed. The same testicular measurements calculated with different formulas $(\mathrm{V}=$ $\mathrm{L} \times \mathrm{W} \times \mathrm{H} \times 0.71$ vs. $\mathrm{V}=\mathrm{L} \times \mathrm{W} \times \mathrm{H} \times 0.52)$ produced $26.76 \%$ difference in the value of testicular volume.

\section{Discussion}

Before promulgation of the guidelines and recommendations for the use of formula $\mathrm{V}=\mathrm{L} \times \mathrm{W} \times \mathrm{H} \times 0.71$ for the testicular volume measurement in 2020, many studies had shown that the formula $\mathrm{V}=\mathrm{L} \times \mathrm{W} \times \mathrm{H} \times 0.71$ is more accurate since 2000; however, some manufactures of ultrasound imaging systems had not adopted $i^{(6-10,14-17)}$. In our study population, the overall conformity rate was $29.65 \%$, the data acquired by some ultrasound systems do not meet the value calculated with reference to the formula by the updated guidelines. The values of testicular volume obtained by the formulas adopted by different ultrasound systems caused a discrepancy of $26.76 \%$. While they are concordant with the value calculated according to the formula that $\mathrm{V}=\mathrm{L} \times \mathrm{W} \times \mathrm{H} \times 0.52$. Obviously, some of the ultrasound systems had adopted formula $\mathrm{V}=\mathrm{L} \times \mathrm{W} \times \mathrm{H} \times 0.52$, other than $\mathrm{V}=\mathrm{L} \times \mathrm{W} \times \mathrm{H} \times 0.71$. We believe that the reason is that some manufactures have not adopted the updated research result, and failed to update the related parameters or the formula. The discrepancy of $26.76 \%$ is detrimental in some cases, leading to an incorrect interpretation and a subsequent bias. For example, if two testicular volumes obtained by an ultrasound system with a built-in formula that $\mathrm{V}=\mathrm{L} \times \mathrm{W} \times \mathrm{H} \times 0.52$ are $26.3 \mathrm{~mL}(13.0 \mathrm{~mL}$ and $13.3 \mathrm{~mL}$, respectively), the urologist will determine that the two testes are not in normal size, with reference that the low size of a normal testis is $14 \mathrm{~mL}$, and both are $28 \mathrm{~mL}$ (these references were obtained according to the formula that $\mathrm{V}=\mathrm{L} \times \mathrm{W} \times \mathrm{H} \times 0.71)$. However, according to the latest guidelines, the testicular volumes should be $33.34 \mathrm{~mL}$ (16.48 $\mathrm{mL}$ and $16.86 \mathrm{~mL}$, respectively), so these values meet the normal criteria.

During the previous studies on the measurement of testicular volume, several formulas have been invented, such as
$\mathrm{V}=\mathrm{L} \times \mathrm{W} \times \mathrm{H} \times .52, \mathrm{~V}=\mathrm{L} \times \mathrm{W}(2) \times 0.52, \mathrm{~V}=\mathrm{L} \times \mathrm{W}(2) \times 0.59$, and $\mathrm{V}=\mathrm{L} \times \mathrm{W} \times \mathrm{H} \times 0.71$; among them, $\mathrm{V}=\mathrm{L} \times \mathrm{W} \times \mathrm{H} \times$ 0.71 has been proved the most accurate ${ }^{(6-10,14-17)}$. Bahk et al., and Takihara et al. reported that the normal range of adult testicular volume (calculated by $\mathrm{V}=\mathrm{L} \times \mathrm{W} \times \mathrm{H} \times 0.71$ ) was greater than $14 \mathrm{~mL}$ in Japan, greater than $17 \mathrm{~mL}$ in the United States, and around $18 \mathrm{~mL}$ in the South Korea ${ }^{(2,5)}$. There is still no official reference for normal testicular size, but accurate assessment is significant for an infertile male. Studies have shown that the testis size varies with racial, regional, body weight, height, and nourishment conditions $^{(5,18)}$. Therefore, comprehensive evaluation combining the measurement of testicular volume with other parameters may be more reasonable in the clinical practice of reproductive medicine $\mathrm{e}^{(5,18,19)}$.

The limitations of this study are that only six ultrasound systems by five manufactures were investigated, and the sample was small.

The findings of this study suggest that the manufacturers of ultrasound imaging systems should update related parameters in time, and the sonographers, radiologists, and urologists should be aware of the fact that some ultrasound systems have not adopted the proper formula for the assessment of testicular volume, which produces discrepancy in clinical practice, and the testicular volume needs to be calculated using a more reliable updated formula.

\section{Conclusion}

In conclusion, the values of testicular volume obtained by some ultrasound systems are not accurate according to the formula recommended by the updated guidelines. Sonographers, radiologists and urologists should validate the testicular volume with reference to the original measurements and the updated formula.

\section{Conflict of interest}

The authors do not report any financial or personal connections with other persons or organizations, which might negatively affect the contents of this publication and/or claim authorship rights to this publication. 


\section{References}

1. Sakamoto H, Yajima T, Nagata M, Okumura T, Suzuki K, Ogawa Y: Relationship between testicular size by ultrasonography and testicular function: measurement of testicular length, width, and depth in patients with infertility. Int J Urol 2008; 15: 529-533.

2. Bahk JY, Jung JH, Jin LM, Min SK: Cut-off value of testes volume in young adults and correlation among testes volume, body mass index, hormonal level, and seminal profiles. Urology 2010; 75: 1318-1323.

3. Tyloch JF, Wieczorek AP: Standards for scrotal ultrasonography. J Ultrason 2016; 16: 391-403.

4. Takihara H, Sakatoku J, Fujii M, Nasu T, Cosentino MJ, Cockett AT: Significance of testicular size measurement in andrology. I. A new orchiometer and its clinical application. Fertil Steril 1983; 39: 836-840.

5. Behre HM, Nashan D, Nieschlag E: Objective measurement of testicular volume by ultrasonography: evaluation of the technique and comparison with orchidometer estimates. Int J Androl 1989; 12: 395-403.

6. Sakamoto H, Saito K, Oohta M, Inoue K, Ogawa Y, Yoshida H: Testicular volume measurement: comparison of ultrasonography, orchidometry, and water displacement. Urology 2007; 69: 152-157.

7. Sakamoto H, Saito K, Ogawa Y, Yoshida H: Testicular volume measurements using Prader orchidometer versus ultrasonography in patients with infertility. Urology 2007; 69: 158-162.

8. Mbaeri TU, Orakwe JC, Nwofor AM, Oranusi CK, Mbonu OO: Ultrasound measurements of testicular volume: comparing the three common formulas with the true testicular volume determined by water displacement. Afr J Urol 2013; 19: 69-73.

9. Kuijper EA, van Kooten J, Verbeke JI, van Rooijen M, Lambalk CB: U1trasonographically measured testicular volumes in 0 - to 6-year-old boys. Hum Reprod 2008; 23: 792-796.

10. Diamond DA, Paltiel HJ, DiCanzio J, Zurakowski D, Bauer SB, Atala A et al.: Comparative assessment of pediatric testicular volume: orchidometer versus ultrasound. J Urol 2000; 164: 1111-1114.
11. Sotos JF, Tokar NJ: A medical calculator to determine testicular volumes matching ultrasound values from the width of the testis obtained in the scrotum with a centimeter ruler. Int J Pediatr Endocrinol 2017; 2017: 14.

12. Lin CC, Huang WJ, Chen KK: Measurement of testicular volume in smaller testes: how accurate is the conventional orchidometer? J Androl 2009; 30: 685-689.

13. Goede J, Hack WW, Sijstermans K, van der Voort-Doedens LM, Van der Ploeg T, Meij-de Vries A et al.: Normative values for testicular volume measured by ultrasonography in a normal population from infancy to adolescence. Horm Res Paediatr 2011; 76: 56-64.

14. Freeman S, Bertolotto M, Richenberg J, Belfield J, Dogra V, Huang DY et al.: Ultrasound evaluation of varicoceles: guidelines and recommendations of the European Society of Urogenital Radiology Scrotal and Penile Imaging Working Group (ESUR-SPIWG) for detection, classification, and grading. Eur Radiol 2020; 30: 11-25.

15. Hsieh ML, Huang ST, Huang HC, Chen Y, Hsu YC: The reliability of ultrasonographic measurements for testicular volume assessment: comparison of three common formulas with true testicular volume. Asian J Androl 2009; 11: 261-265.

16. Raza SA, Jhaveri KS: Imaging in male infertility. Radiol Clin North Am 2012; 50: 1183-1200.

17. Guideline developed in collaboration with the American College of Radiology; Society for Pediatric Radiology; Society of Radiologists in Ultrasound: AIUM practice guideline for the performance of scrotal ultrasound examinations. J Ultrasound Med 2015; 34: 1-5.

18. Dave DS, Tiwari VD: Normal testicular volume in school children of low socio-economic group. Indian Pediatr 1983; 20: 901-905.

19. Ku JH, Kim ME, Jeon YS, Lee NK, Park YH: Factors influencing testicular volume in young men: results of a community-based survey. BJU Int 2002; 90: 446-450. 